\title{
Simulation thermomécanique du soudage par friction-malaxage
}

\author{
Eric Feulvarch*,** — Frédéric Boitout* \\ Jean-Michel Bergheau** \\ * ESI Group \\ 70 rue Robert \\ F-69458 Lyon cedex 06 \\ eric.feulvarch@esi-group.com \\ ** LTDS UMR 5513 CNRS/ECL/ENISE \\ 58 rue Jean Parot \\ F-42023 Saint-Etienne cedex 02
}

RÉSUMÉ. Le soudage par friction-malaxage est un procédé de soudage sans apport de matière où la source de chaleur provient intégralement du malaxage du matériau à souder, ainsi que de son frottement sur l'outil. Dans cet article, un modèle élément fini est proposé pour simuler le couplage thermomécanique durant la phase stationnaire du procédé. Les équations de conservation de l'énergie, de la masse et de la quantité de mouvement sont traitées à partir d'un élément fini tétraédrique. Un exemple de simulation est présenté pour un alliage d'aluminium de type 7075.

ABSTRACT. Friction Stir Welding is a welding process where the heat generation is provided by the mechanical dissipation due to the deformations and the friction between the tool and the sheets. This paper describes a finite element model to simulate the heating phenomenon during the steady-state of the process. The stress equilibrium, the energy conservation and the mass conservation are studied in a fully coupled model using a tetrahedral finite element. An example is presented for an aluminium alloy 7075.

MOTS-CLÉS : soudage par friction-malaxage, éléments finis, couplage thermomécanique.

KEYWORDS: friction stir welding, finite element, thermomechanical coupling.

DOI:10.3166/REMN.16.865-887 (C) 2007 Lavoisier, Paris

REMN - 16/2007. Modélisations numériques en mécanique, pages 865 à 887 


\section{Introduction}

Le soudage est un procédé d'assemblage couramment mis en œuvre dans le milieu industriel à partir, par exemple, des techniques de type soudage au plasma, au laser, par faisceau d'électrons ou par résistance. Comme pour les méthodes de soudage par friction plus conventionnelles utilisées depuis le début des années 1950, la soudure par friction-malaxage est réalisée en phase solide sans apport de matière. Les applications actuelles de ce procédé portent essentiellement sur les alliages d'aluminium et, en particulier, ceux réputés «difficilement soudables ». En effet, l'expérience a montré que le fait de souder sans atteindre la fusion permet au procédé Friction Stir Welding d'assembler ce type d'alliages.

Toutefois, comme tout procédé de soudage, le soudage FSW appliqué aux alliages induit des modifications microstructurales, des contraintes résiduelles et des distorsions difficiles à maîtriser. L'ensemble de ces phénomènes peut être responsable d'une détérioration des propriétés d'usage, aussi bien en termes de géométrie et qualité d'assemblage qu'en termes de résistance et tenue en fatigue. En effet, des contraintes résiduelles élevées peuvent conduire à la fragilisation du joint ou encore, des distorsions peuvent mener à des défauts géométriques inacceptables du point de vue de la conception.

La modélisation numérique d'un tel procédé est un problème complexe dans lequel interviennent des couplages physiques forts, en particulier, entre la mécanique et la thermique puisque les élévations de température ne sont dues qu'à la dissipation mécanique. Le malaxage important du matériau de base conduit à des niveaux de déformation et de taux de déformation très importants à l'état solide. C'est principalement la raison pour laquelle il semble difficile de simuler ce procédé à partir de modèles numériques dédiés à la modélisation des procédés plus conventionnels (ex. TIG, MIG...) où la température de fusion est atteinte. Pour ces derniers, les déformations sont souvent faibles dans la phase solide et seule la déformation thermique est prise en compte dans la zone fondue.

L'objectif des travaux présentés dans cet article est de développer un modèle élément fini dédié à la simulation de l'écoulement thermomécanique au cours du soudage FSW. La première section, essentiellement bibliographique, est consacrée au soudage FSW dans sa globalité. Le modèle numérique qui est ensuite décrit permet de modéliser l'apport de chaleur à partir de l'écoulement matériel. Le couplage thermomécanique est traité à partir d'un formalisme eulérien en considérant uniquement la phase stationnaire du procédé. Pour cela, un élément fini tétraédrique linéaire de type $\mathrm{P} 1+/ \mathrm{P} 1$ a été développé dans le code de calcul SYSWELD ${ }^{\circledR}$ dédié à la simulation des procédés de soudage et de traitement thermique (SYSWELD $\AA$, 2006). Dans la dernière section, un exemple de soudage est présenté pour un alliage à durcissement structural de type 7075 . 


\section{Le procédé FSW}

\subsection{Principe du soudage FSW}

Le soudage par friction-malaxage ou «Friction Stir Welding» est un procédé récent développé au sein de l'institut de soudure britannique (The Welding Institute) au début des années 1990 (Shercliff et Colegrove, 2002). Le soudage FSW est un procédé sans apport de matière pour lequel, les échauffements proviennent uniquement de la dissipation induite par le malaxage du matériau de base et de son frottement sur l'outil.

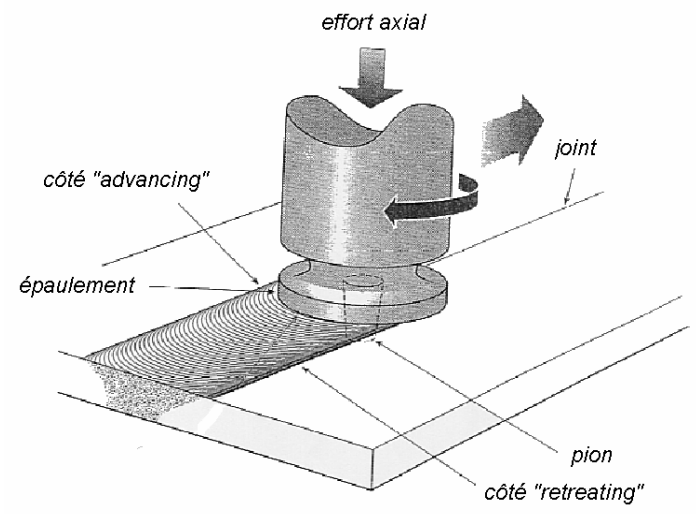

Figure 1. Principe du soudage par Friction Stir Welding

Ce dernier est entraîné en rotation et en translation le long du joint à souder comme le montre la figure 1 pour le soudage «bout-à-bout» de deux tôles minces. L'outil est constitué d'un épaulement et d'un pion. La vitesse de rotation est généralement comprise entre 100 et $1500 \mathrm{tr} \cdot \mathrm{min}^{-1}$ et la vitesse d'avance peut atteindre $1500 \mathrm{~mm} \cdot \mathrm{min}^{-1}$. L'effort axial de maintien de l'outil contre les faces supérieures des tôles à assembler varie quant à lui de quelques $\mathrm{kN}$ à quelques dizaines de $\mathrm{kN}$ lorsque les épaisseurs à souder deviennent importantes (de l'ordre de $50 \mathrm{~mm}$ ).

En général, les températures atteintes durant le soudage ne dépassent pas $80 \%$ de la température de fusion du métal de base. Par ailleurs, on distingue deux zones de part et d'autre du joint soudé : le côté "retreating» et le coté "advancing» caractéristiques de l'asymétrie du soudage FSW.

\subsection{Le joint soudé}

Le joint obtenu par soudage FSW d'un alliage d'aluminium présente 4 zones distinctes comme le montre la figure 2 pour un alliage de type 2024 : 
- le métal de base qui correspond à la partie de la pièce où la température a été suffisamment faible pour ne pas entraîner de changements significatifs de la microstructure,

- une zone affectée thermiquement qui ne présente que de faibles transformations microstructurales. Les déformations mécaniques y sont très faibles,

- une zone affectée thermomécaniquement qui a subi des déformations mécaniques d'un niveau bien plus important que dans la ZAT (désorientation importante de la microstructure). La température y a été suffisamment élevée pour entraîner des modifications microstructurales de façon significative,

- le noyau qui est une zone de forme elliptique dans laquelle apparaît une microstructure allongée en bande concentrique (« onion rings »). Les déformations y sont maximales et la frontière entre le noyau et la ZATM est facilement identifiable.

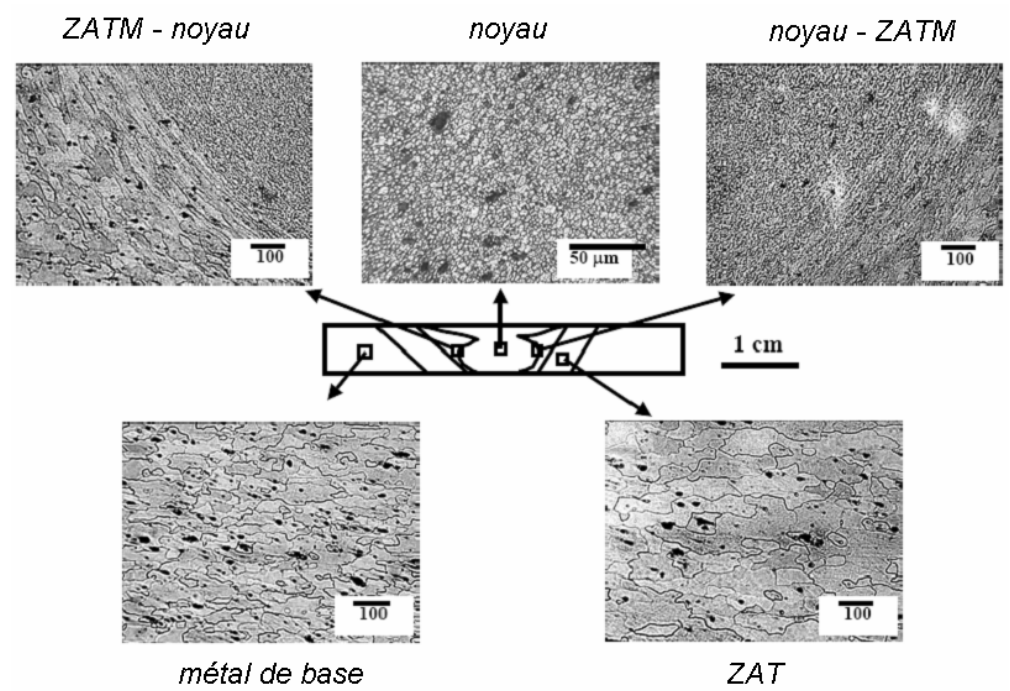

Figure 2. Microstructure d'un joint soudé pour alliage d'aluminium de type 2024 (Connolly et al., 2004)

La microstructure du joint soudé a fait l'objet de nombreuses études dans la littérature. Ces travaux portent essentiellement sur l'état de la recristallisation et sur les mécanismes de précipitation. Tous ces mécanismes sont étroitement liés au profil de dureté du matériau au travers du joint soudé. Des profils typiques de dureté sont représentés sur la figure 3 .

Pour l'alliage de la série 5XXX écroui, une diminution faible de la dureté apparaît tout d'abord dans la ZAT. Elle provient de la restauration. Ce phénomène est caractérisé par la recombinaison et le réarrangement des dislocations conduisant 
à une légère diminution de leur densité. Dans le même temps, la recristallisation prend place au voisinage de la ZATM d'où une diminution significative de la dureté. Pour le même alliage recuit, la légère augmentation de la dureté dans le noyau semble provenir de l'affinement de la structure cristalline.

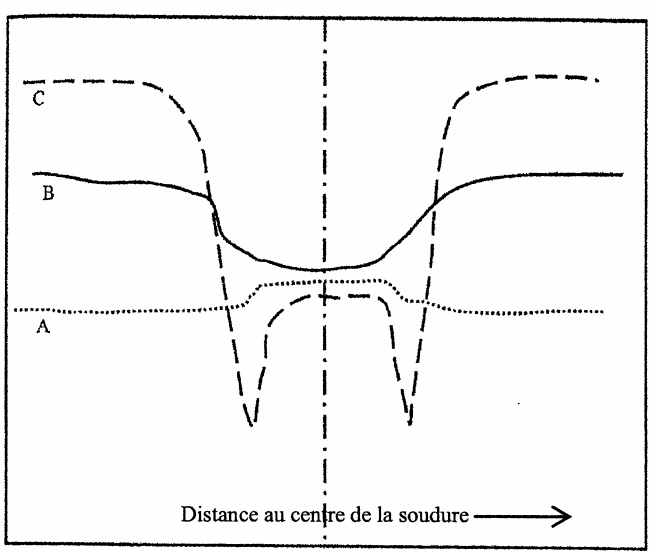

Figure 3. Représentation schématique des profils de dureté pour différents types d'alliages d'aluminium: $A-5 X X X$ recuit, $B$ - $5 X X X$ écroui et $C$ - alliage à durcissement structural (Reynolds, 2003)

Pour l'alliage à durcissement structural préalablement revenu, la température augmentant au voisinage du centre de la soudure, on observe dans la ZAT une croissance et remise en solution complète de la précipitation durcissante initiale liées à un survieillissement. Cela conduit à un minimum de dureté très marqué à proximité de la ZATM. Dans le noyau, un niveau de dureté plus important peut être retrouvé. Il atteint parfois celui du métal de base. Cela s'explique principalement par les fortes déformations vécues induisant une recristallisation dynamique et donc un affinement de la microstructure. Il peut également se produire un phénomène de maturation causé par le refroidissement rapide de la solution solide (Gallais, 2005).

\subsection{Les conséquences mécaniques induites}

Les procédés de soudage engendrent généralement la formation de distorsions et de contraintes résiduelles qui dépendent de l'histoire thermique vécue par le joint au cours du procédé. Elles proviennent de l'évolution fortement non homogène de la masse volumique qui s'explique principalement par : 
- la dilatation thermique,

- la variation éventuelle de la compacité des constituants en présence.

Le second phénomène est couramment négligé pour modéliser le soudage des alliages d'aluminium en supposant que la masse volumique est exclusivement thermodépendante. Ce n'est pas le cas pour les aciers. Pour un acier fer-carbone, la structure cristalline de la phase $\alpha$ est cubique centrée alors que celle de la phase $\gamma$ est cubique à face centrée. Par conséquent, la masse volumique peut présenter de fortes variations qui dépendent des constituants présents à chaque instant dans les différentes zones de la soudure et dont les proportions résultent de l'histoire thermique vécue (figure 4).

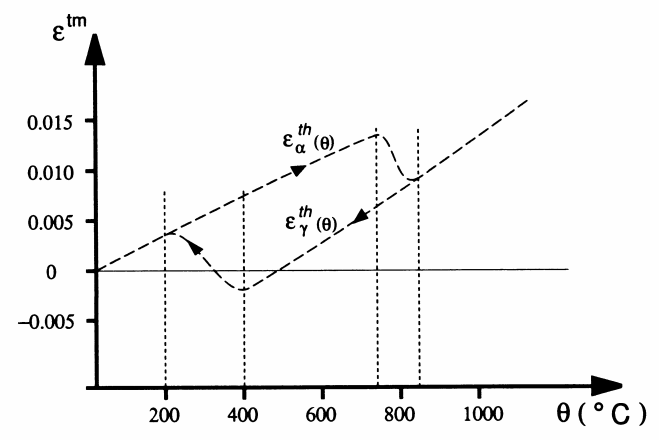

Figure 4. Essai de dilatométrie libre

La formation des effets mécaniques résiduels peut être accentuée par les conditions de bridage empêchant la déformation des éléments soudés lors du retrait thermique au refroidissement. Les distorsions typiquement rencontrées pour le soudage « bout à bout » de tôles minces sont schématisées sur la figure 5 .

Dans cette configuration, les contractions longitudinales et transversales proviennent principalement du retrait thermique lors du refroidissement de la zone fondue dont la forme en $\mathrm{V}$ peut provoquer un gauchissement et un fléchissement relativement importants. Ces distorsions sont fréquemment rencontrées pour des procédés de soudage conventionnels où la fusion est atteinte. Elles tendent à diminuer lorsque l'épaisseur des tôles augmente, au détriment d'une augmentation des contraintes résiduelles.

Concernant le soudage FSW, peu d'analyses sont décrites dans la littérature. Les faibles niveaux de température atteints semblent conduire à des valeurs de contraintes résiduelles relativement basses comparativement aux procédés par fusion. De plus, il apparaît que les contraintes résiduelles maximales se situent dans la direction longitudinale et ne dépassent pas $50 \%$ de la limite d'élasticité du 
matériau de base. Elles peuvent tout de même être suffisamment élevées pour affecter la tenue à la fatigue du joint soudé (Donne et al., 2001).
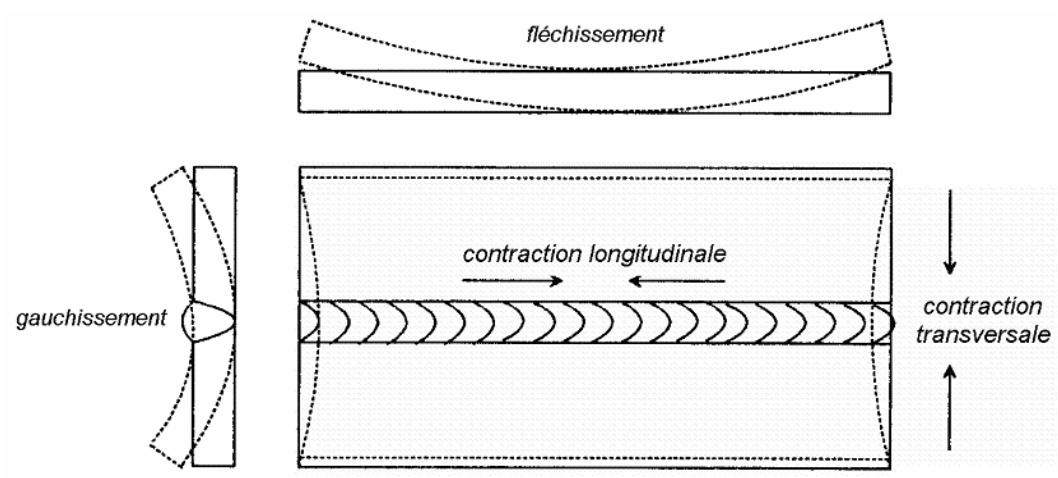

Figure 5. Distorsions typiquement rencontrées lors du soudage en $V$ de deux tôles minces assemblées en "bout à bout "

\section{Modélisation thermomécanique du procédé FSW}

\subsection{Introduction}

Le but de cette étude étant de modéliser l'apport de chaleur, il est indispensable de prendre en compte les aspects thermiques et mécaniques au travers de la conservation de la quantité de mouvement, de la masse et de l'énergie.

De manière générale, les procédés de soudage mettent en jeu une source de chaleur mobile de très petite taille comparée à celle de la structure étudiée. La modélisation locale des effets mécaniques, thermiques et métallurgiques nécessite des maillages très fins au voisinage de la source pour conduire une analyse éléments finis. Un raffinement important du joint peut conduire à des tailles de problème très importantes. Cela peut être évité à partir d'une procédure de remaillage en ne raffinant la discrétisation qu'au voisinage de la source de chaleur le long du joint à souder. Ce type d'analyses transitoires conduit tout de même à des temps de calcul importants et très souvent, on admet qu'un régime permanent s'établit lorsque la structure soudée présente une géométrie de translation sur des longueurs importantes.

Concernant le soudage FSW, la longueur des joints soudés peut atteindre plusieurs mètres. Pour déterminer le champ de température pendant la phase stationnaire du procédé, il suffit de réaliser une analyse stationnaire en se plaçant dans un référentiel lié à la source de chaleur. C'est la raison pour laquelle, nous avons opté pour un formalisme eulérien (Bastier et al., 2005). 


\subsection{Lois de conservation}

L'équilibre thermomécanique est régi par la conservation de l'énergie, la conservation de la quantité de mouvement et la conservation de la masse :

$$
\begin{aligned}
& \alpha \cdot \overline{\bar{S}}: \overline{\bar{D}}+\operatorname{div}(\vec{q})=\rho \cdot C \cdot \vec{v} \cdot \overrightarrow{\operatorname{grad}}(\theta) \\
& \overrightarrow{\operatorname{div}}(\overline{\bar{S}})-\overrightarrow{\operatorname{grad}}(p)=\overrightarrow{0} \\
& \operatorname{div}(\vec{v})=0
\end{aligned}
$$

où $\theta$ représente la température, $\vec{v}$ la vitesse, $\vec{q}$ le vecteur courant de chaleur, $p$ la pression hydrostatique et $\overline{\bar{S}}$, le déviateur des contraintes de Cauchy dans la configuration eulérienne. $\rho$ correspond à la masse volumique et $C$, la chaleur spécifique. $\alpha$ est un coefficient variant de 0.9 à 1 lorsque la puissance mécanique $\overline{\bar{S}}: \overline{\bar{D}}$ n'est pas intégralement dissipée sous forme de chaleur (prise en compte d'un éventuel écrouissage) (Fourment et al., 2004). $\overline{\bar{D}}$ est le tenseur des taux de déformation eulériens défini à partir du champ des vitesses comme suit :

$$
\overline{\bar{D}}=\frac{1}{2} \cdot\left(\overline{\overline{\operatorname{grad}}}(\vec{v})+\overline{\overline{\operatorname{grad}}}^{t}(\vec{v})\right)
$$

L'ensemble des modélisations présentées dans la littérature s'accordent sur le fait que le poids propre de la structure et les efforts d'inertie sont négligeables au regard de l'importance des efforts internes dans les tôles soudées. Par ailleurs, l'objectif de cette modélisation étant de simuler la phase de soudage et non pas d'accéder aux conséquences mécaniques du procédé, l'effet de la dilatation thermique est supposé négligeable sur l'écoulement matériel.

\subsection{Comportements thermique et mécanique}

Le gradient des températures est relié au vecteur courant de chaleur à partir de la loi de Fourier :

$$
\vec{q}=-\lambda \overrightarrow{\operatorname{grad}}(\theta)
$$

où $\lambda$ est la conductivité thermique. 
Pour le comportement mécanique, il est relativement difficile de tenir compte d'un effet d'histoire dans une modélisation stationnaire c'est-à-dire figée. La majorité des modèles éléments finis existants reposent soit sur la connaissance $a$ priori des trajectoires suivies par la matière (Nguyen et Rahamian, 1981 ; Dang Van et al., 1985 ; Maitournam, 1989; Bergheau et al., 1991) ou sur l'hypothèse des petites déformations (Rajadhyaksha et Michaleris, 2000 ; Shanghvi et Michaleris, 2002; Zhang et Michaleris, 2004). Pour le procédé FSW, les niveaux de déformations et de taux de déformation sont très élevés. De plus, la microstructure évolue de façon très significative à l'état solide (recristallisation dynamique...). Par conséquent, il semble relativement complexe de modéliser l'élasticité du matériau et un éventuel écrouissage. Shercliff et al., proposent de modéliser l'écoulement en adoptant une loi de comportement ne présentant aucune sensibilité de la contrainte au niveau de déformation atteint (Shercliff et Colegrove, 2002) :

$$
\overline{\bar{S}}=2 \cdot \mu(\overline{\bar{D}}, \theta) \cdot \overline{\bar{D}}
$$

Cette loi de comportement de type fluide visqueux incompressible fait apparaitre la viscosité dynamique $\mu$ définie à partir de la loi de Norton-Hoff couramment mise en œuvre pour modéliser les procédés de mise en forme à chaud. Elle fait intervenir la consistance $K$ du matériau de base, ainsi qu'une sensibilité $m$ de la contrainte à la vitesse de déformation :

$$
\mu=K(\theta) \cdot(\sqrt{3} \cdot D)^{m(\theta)-1}
$$

où $D$ représente le taux de déformation équivalent :

$$
D=\sqrt{\frac{2}{3} \overline{\bar{D}}: \overline{\bar{D}}}
$$

Il apparaît clairement que lorsque $D$ tend vers 0 avec $0<m<1, \mu$ tend vers l'infini. Ce cas de figure ne peut pas être traité numériquement par la méthode des éléments finis. C'est la raison pour laquelle, Wagoner et Chenot (2001) proposent d'introduire un paramètre $\varepsilon$ faible en définissant une loi équivalente régularisée :

$$
\mu^{*}=K(\theta) \cdot\left(3 \cdot D^{2}+3 \cdot \varepsilon^{2}\right)^{\frac{m(\theta)-1}{2}}
$$

\subsection{Conditions aux limites}

La modélisation du contact outil-matière constitue très certainement la partie la plus complexe de la modélisation. Il existe peu de données le concernant. Les 
modélisations présentées dans la littérature reposent principalement sur l'imposition d'une valeur de contrainte tangentielle à l'interface (Colegrove et Shercliff, 2004) :

$$
\vec{\tau}=\tau \cdot \frac{\Delta \vec{v}}{\|\Delta \vec{v}\|}
$$

où $\vec{\tau}$ représente la contrainte tangentielle et $\Delta \vec{v}$, la vitesse de glissement.

Par ailleurs, les interactions thermiques entre l'outil et le matériau de base sont négligés en supposant que la force exercée par l'outil au niveau de son épaulement conduit à un contact suffisamment intime pour que le différentiel de température à l'interface soit très faible (Fourment et al., 2004). De cette façon, seule la dissipation mécanique $\vec{\tau} . \Delta \vec{v}$ intervient dans l'expression des conditions aux limites thermiques au niveau du contact outil-matière (figure 6) :

$$
q=-\vec{q} \cdot \vec{n}=\beta \cdot \vec{\tau} \cdot \Delta \vec{v}
$$

$q$ correspond à la densité de flux de chaleur reçu et $\beta$ est la proportion de la puissance dissipée reçue par les tôles à souder sachant que l'outil absorbe la proportion $1-\beta$. La valeur de $\beta$ est généralement définie à partir des effusivités $e$ des matériaux en contact:

$$
\beta=\frac{e^{\text {matériau }}}{e^{\text {matériau }}+e^{\text {outil }}} \text { avec } e=\sqrt{\lambda \cdot \rho \cdot C}
$$

Cette expression est issue de l'analyse d'un contact dissipatif entre deux milieux semi-infinis. Il est important de noter que des études récentes ont mis en évidence la complexité du partage de la puissance dissipée au niveau d'une interface, en particulier, dans le cas du contact électrothermique où la puissance est dissipée par effet Joule (Le Meur, 2002 ; Feulvarch et al., 2004).

Concernant les échanges entre les tôles et l'air environnant, la densité de flux thermique est habituellement modélisée à partir d'un coefficient d'échange par convection $h_{\text {ext }}$ comme suit :

$$
q=h_{e x t} \cdot\left(\theta_{\text {ext }}-\theta\right)
$$

$\theta_{\text {ext }}$ représente la température de l'air environnant.

De la même manière, la modélisation du contact thermique entre les tôles et le support de soudage se fait à partir d'un coefficient d'échange $h_{\text {contact }}$ : 


$$
q=h_{\text {contact }} \cdot\left(\theta_{\text {support }}-\theta\right)
$$

$\theta_{\text {support }}$ est la température sur la surface du support de soudage.

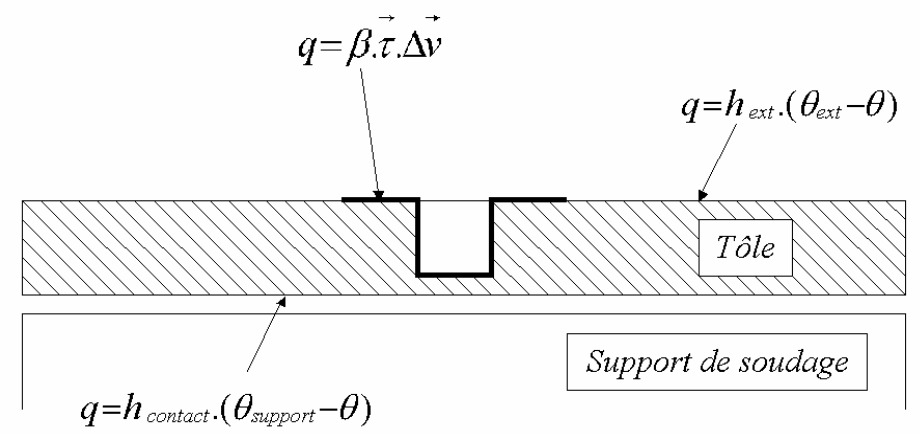

Figure 6. Conditions aux limites thermiques

\section{Modélisation numérique}

Etant donné le couplage thermomécanique qu'implique la modélisation du soudage FSW, l'analyse thermique et l'analyse mécanique doivent être menées conjointement. En effet, la dissipation mécanique, les phénomènes de transport convectif et la thermodépendance du comportement mécanique impliquent un couplage physique fort nécessitant la mise en œuvre de méthodes numériques spécifiques.

\subsection{Elément fini P1+/P1}

Pour modéliser l'écoulement visqueux incompressible, la modélisation par la méthode des éléments finis se heurte à une difficulté de nature «numérique ». La discrétisation à mettre en œuvre doit être choisie de manière judicieuse pour ne pas faire apparaître de phénomène de verrouillage («locking») se traduisant par un champ de vitesse aberrant. D’un point de vue mathématique, ceci peut s'expliquer par le fait que le champ des vitesses doit être suffisamment « riche » pour satisfaire à la fois l'équilibre des efforts et l'incompressibilité.

Depuis le début des années 1960, beaucoup de travaux ont été consacrés à ce problème. Il apparaît que la contrainte d'incompressibilité peut être traitée par des méthodes de projection décrites par (Gresho et Sani, 2000) ou par une méthode de pénalisation qui nécessite la mise en œuvre de techniques numériques du type intégration réduite ou intégration sélective (Zienkiewicz et Taylor, 1990 ; Reddy et 
Gartling, 2000). L'incompressibilité peut également être prise en compte par la méthode des multiplicateurs de Lagrange ou par la méthode du lagrangien augmenté en introduisant des inconnues supplémentaires comme, par exemple, la pression (Bathe, 1996). Dans ce cas, une attention particulière doit être apportée au choix de la discrétisation à mettre en œuvre pour satisfaire la condition de (Babuska, 1973 ; Brezzi, 1974).

L'élément fini P1+/P1 est un élément très répandu pour la modélisation des procédés de mise en forme à chaud (figure 7) (Arnold, 1984 ; Jaouen, 1998). Pour cet élément tétraédrique, le champ discret des pressions $p^{h}$ est linéaire et continu (P1). L'exposant + signifie qu'un nœud est ajouté pour l'interpolation de la vitesse $\rightarrow H$ $\vec{v}(\mathrm{P} 1+)$ de sorte que :

$$
\vec{v}^{H}=\vec{v}^{h} \oplus \vec{v}^{b}
$$

où $\vec{v}^{h}$ représente la partie linéaire $(\mathrm{P} 1)$ et $\vec{v}$, la partie « bulle » du champ des$$
\rightarrow b
$$
vitesses.

Le nœud interne est situé au centre de l'élément. La fonction d'interpolation « bulle » correspond à un raffinement de type $h$ c'est-à-dire qu'elle est de degré 1 par morceau à l'intérieur du tétraèdre.

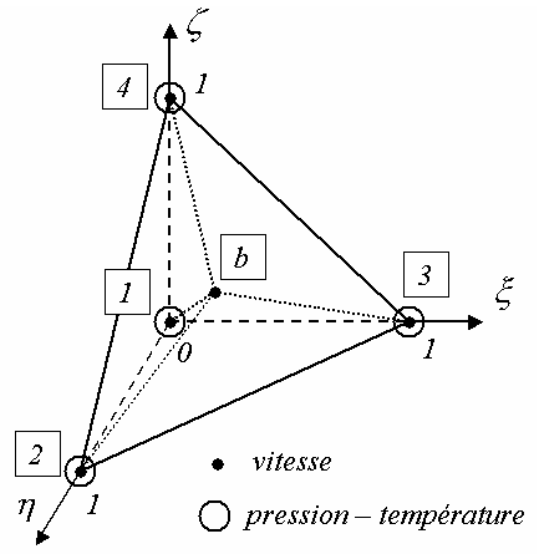

Figure 7. Tétraèdre $P 1+/ P 1$ 


\subsection{Discrétisation et résolution}

Pour appliquer la méthode des éléments finis, la formulation intégrale faible du problème couplé thermomécanique s'écrit sous forme discrète de la manière suivante :

$$
\begin{aligned}
& \forall \vartheta^{h}, \int_{\Omega} \overrightarrow{\operatorname{grad}}\left(\vartheta^{h}\right) \cdot \lambda \cdot \overrightarrow{\operatorname{grad}}\left(\theta^{h}\right) \cdot d v+\int_{\Omega} \phi^{h} \cdot \rho \cdot C \cdot \vec{v}^{H} \cdot \overrightarrow{\operatorname{grad}}\left(\theta^{h}\right) \cdot d v \\
& \quad-\int_{\Omega} \phi^{h} \cdot \alpha \cdot \overline{\bar{S}}: \overline{\bar{D}} \cdot d v-\int_{\partial \Omega} \vartheta^{h} \cdot q \cdot d s=0 \\
& \forall \vec{w}^{H}, \int_{\Omega} \overline{\bar{S}}\left(\vec{v}^{H}\right): \overline{\bar{D}}\left(\vec{w}^{H}\right) \cdot d v-\int_{\Omega} p h \cdot \operatorname{div}\left(\vec{w}^{H}\right) \cdot d v-\int_{\partial \Omega} \vec{w}^{H} \vec{T} \cdot d s=0 \\
& \forall q^{h}, \int_{\Omega} q^{h} \cdot \operatorname{div}\left(\vec{v}^{H}\right) \cdot d v=0
\end{aligned}
$$

où $\Omega$ représente le domaine d'étude et $\partial \Omega$, sa frontière. $\vec{w}^{H}, \vartheta^{h}$ et $q^{h}$ sont des champs tests discrets construits respectivement de la même manière que $\vec{v}^{H}, \theta^{h}$ et $p^{h}$.

Pour traiter le problème de diffusion-convection thermique, $\phi^{h}$ est défini à partir de la technique SUPG (Brooks et Hughes, 1982 ; Bergheau et Fortunier, 2004) :

$$
\phi^{h}=\vartheta^{h}+\eta \cdot \vec{v} \cdot \overrightarrow{\operatorname{grad}}\left(\vartheta^{h}\right)
$$

où $\phi^{h}$ est, par construction, un champ discontinu sur les frontières des éléments. $\eta$ est un paramètre ajustable propre à la technique SUPG.

L'application de la méthode des éléments finis au problème thermomécanique conduit à résoudre un système d'équations non linéaires du type :

$$
\left\{\begin{array}{l}
\left\{R_{\theta}(\{\theta\},\{\vec{v}\})\right\} \\
\left.\left\{R_{v}(\{\theta\},\{\vec{v}\},\{p\})\right\}\right\} \\
\left\{R_{p}(\{\vec{v}\})\right\}
\end{array}\right\}=\left\{\begin{array}{l}
0 \\
0 \\
0
\end{array}\right\}
$$


$\left\{R_{\theta}\right\},\left\{R_{v}\right\}$ et $\left\{R_{p}\right\}$ sont les résidus relatifs au problème des transferts de chaleur, à l'équilibre des efforts et à l'incompressibilité. $\{\theta\},\{\vec{v}\}$ et $\{p\}$ sont les vecteurs des inconnues nodales.

Pour résoudre ce système d'équations non linéaires par la méthode de NewtonRaphson, diverses approches peuvent être envisagées. Il est, par exemple, possible de le résoudre à partir d'une méthode indirecte (Pascal et al., 2003). Soient $\{\theta\}^{i}$, $\{\vec{v}\}^{i}$ et $\{p\}^{i}$, les solutions obtenues après $i$ itérations, une meilleure solution sera obtenue en résolvant successivement :

$$
\begin{gathered}
\left\{R_{\theta}\left(\{\theta\}^{i},\{\vec{v}\}\right)\right\}+\left[\frac{\partial R_{\theta}}{\partial \theta}\right]^{i} \cdot\left\{\{\theta\}^{i+1}-\{\theta\}^{i}\right\}=\{0\} \\
\left.\left\{\begin{array}{l}
\left\{R_{v}\left(\{\theta\}^{i+1},\{\vec{v}\}^{i},\{p\}^{i}\right)\right\} \\
\left\{R_{p}\left(\{\vec{v}\}^{i}\right)\right\}
\end{array}\right\}+\left[\begin{array}{l}
{\left[\frac{\partial R_{v}}{\partial \vec{v}}\right]} \\
{\left[\frac{\partial R_{p}}{\partial \vec{v}}\right]}
\end{array}\right]\left[\begin{array}{c}
\left.\frac{\partial R_{v}}{\partial p}\right] \\
0
\end{array}\right]\right]^{i} \cdot\left\{\begin{array}{l}
\{\vec{v}\}^{i+1}-\{\vec{v}\}^{i} \\
\{p\}^{i+1}-\{p\}^{i}
\end{array}\right\}=\{0\}
\end{gathered}
$$

Etant donné le couplage thermomécanique fort qu'simplique la simulation du soudage FSW, l'emploi d'une méthode directe semble tout à fait appropriée. Elle permet de prendre en compte les termes de couplage dans l'opérateur tangent de Newton-Raphson en résolvant :

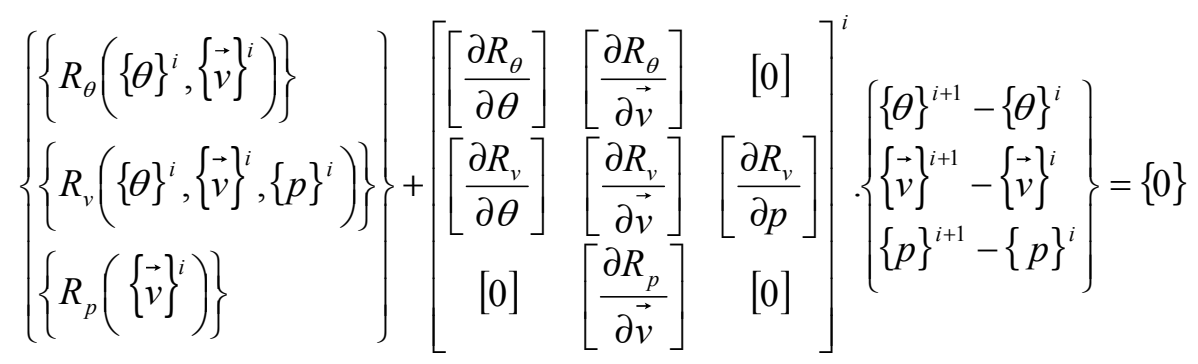

Le processus itératif associé à une méthode directe de résolution converge $a$ priori plus rapidement en nombre d'itérations (Bergheau et Fortunier, 2004).

Il est important de noter que cette dernière équation ne présente que les grandes lignes de l'algorithme de résolution dont les détails sont décrits dans (Feulvarch, 2005): formulation de type «mini-élément», condensation du champ bulle, 
traitement de la loi de comportement par une technique similaire à une méthode de recherche de point fixe, etc.

\section{Application}

\subsection{Présentation du modèle}

Dans cet exemple d'application, 2 tôles d'épaisseur $6 \mathrm{~mm}$ de l'alliage 7075 sont soudées «bout à bout » (figure 8). Le diamètre extérieur de l'épaulement de l'outil est de $22 \mathrm{~mm}$ et son diamètre intérieur mesure $10,2 \mathrm{~mm}$. La géométrie du pion est conique $\left(60^{\circ}\right)$ et sa longueur est de $4,8 \mathrm{~mm}$. L'axe de l'outil est perpendiculaire aux faces supérieures des tôles à assembler.

Dans la littérature, il existe peu de données concernant la consistance $K$ et la sensibilité $m$ à la vitesse de déformation intervenant dans l'expression de la loi de Norton-Hoff sur la plage de température que fait intervenir le soudage FSW. Pour l'alliage 7075, Jin et al. présentent l'évolution de la contrainte d'écoulement lors d'essais de compression pour des vitesses de déformation allant de $0,001 \mathrm{~s}^{-1}$ à $2100 \mathrm{~s}^{-1}$ et des températures comprises entre $23^{\circ} \mathrm{C}$ et $470^{\circ} \mathrm{C}$.
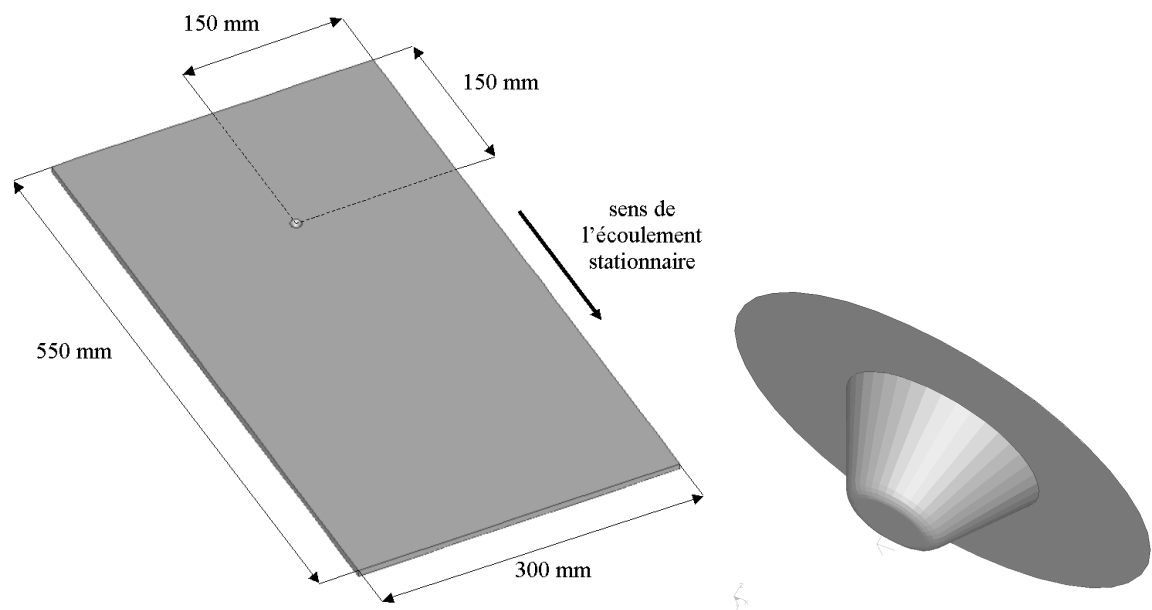

Figure 8. Géométrie et positionnement de l'outil de soudage (Feulvarch, 2005)

Dans cet exemple d'application, la consistance et la sensibilité ont été déterminés à partir des valeurs de la figure 9 en employant une interpolation linéaire pour leur évolution en fonction de la température. 
880 REMN - 16/2007. Modélisations numériques en mécanique

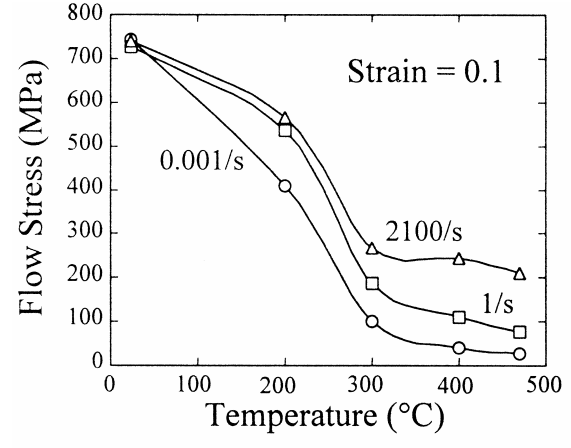

(a)

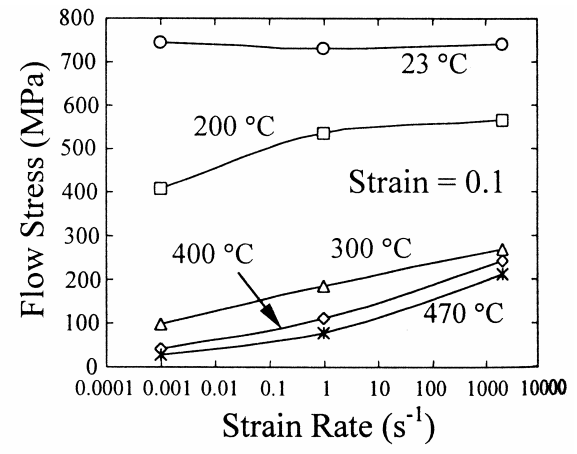

(b)

Figure 9. Evolution de la contrainte d'écoulement en fonction de la température (a) et du taux de déformation (b) pour un alliage de type 7075 (Jin et al., 2000)

La partie surfacique du maillage est présentée sur la figure 10.

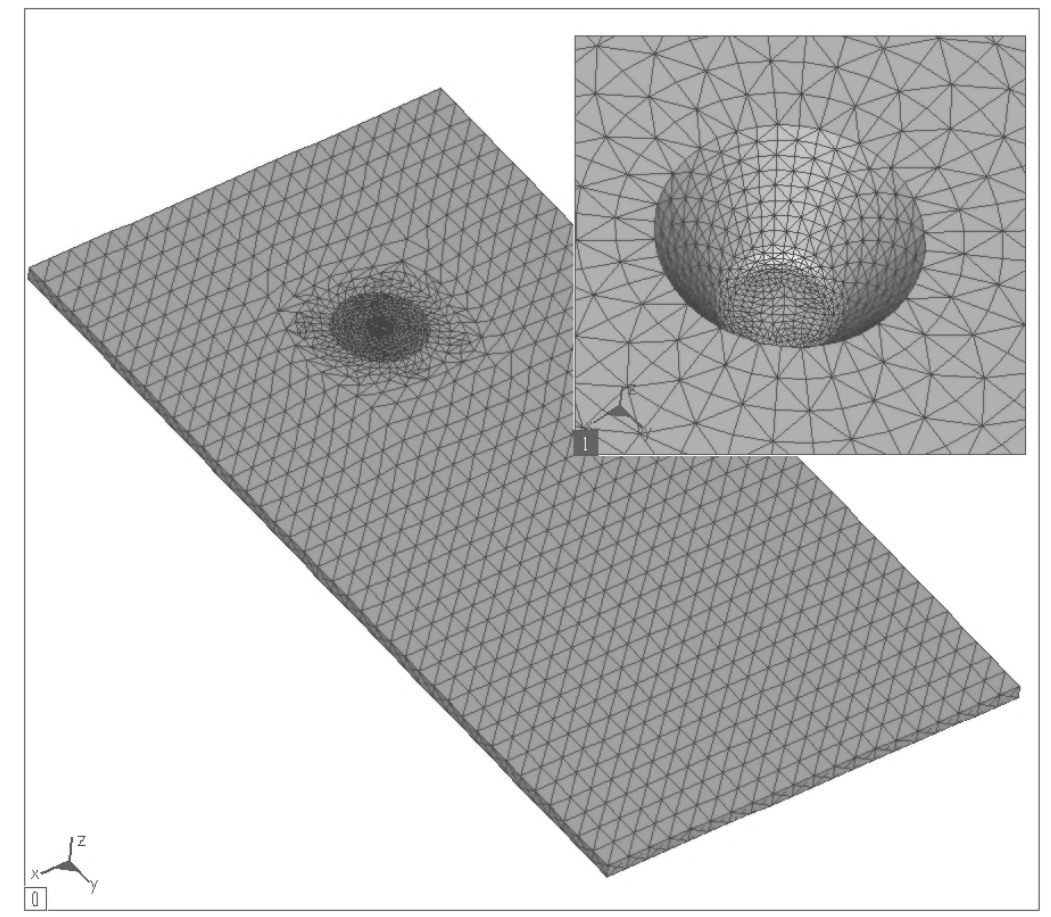

Figure 10. Maillage 
La puissance mécanique est intégralement dissipée sous forme de chaleur $(\alpha=1)[1]$. Les caractéristiques thermiques sont les suivantes (Gallais, 2005) :

- la conductivité thermique $\lambda: 170 \mathrm{~W} \cdot \mathrm{m}^{-1} \cdot \mathrm{K}^{-1}$,

- la densité $\rho: 2400 \mathrm{~kg} \cdot \mathrm{m}^{-3}$,

- la chaleur spécifique $C: 900 \mathrm{~J}^{\mathrm{kg}}{ }^{-1} \cdot \mathrm{K}^{-1}$.

Le coefficient d'échange $h_{\text {ext }}$ est de $30 \mathrm{~W} \cdot \mathrm{m}^{-2} \cdot \mathrm{K}^{-1}$ pour les échanges avec l'air $\left(20^{\circ} \mathrm{C}\right)$. Pour les échanges avec le support de soudage, la valeur de $h_{\text {contact }}$ dépend de la température, de la pression de contact, de la nature des matériaux en contact et de bien d'autres paramètres tels que les états de surface. C'est la raison pour laquelle, la littérature portant sur ce sujet fait apparaître une très large gamme de valeurs. Pour l'exemple proposé, $h_{\text {contact }}$ est pris égal à $350 \mathrm{~W} \cdot \mathrm{m}^{-2} \cdot \mathrm{K}^{-1}$.

\subsection{Modélisation du contact thermique tôle-support}

Pour la modélisation numérique du contact thermique tôle-support, le support est modélisé. La densité de flux de chaleur $q$ est tout d'abord estimée pour chacun des points de Gauss appartenant à chacune des surfaces de contact (Feulvarch et al., 2006) :

$$
\widetilde{q}=h_{\text {contact }} \cdot\left(\widetilde{\theta}_{i}-\theta_{i}\right)
$$

où $\theta_{i}$ représente la température du point de Gauss considéré et $\widetilde{\theta}_{i}$, la température moyenne de la maille opposée (figure 11).

Elément fini de contact opposé à la température moyenne $\widetilde{\theta}_{i}$

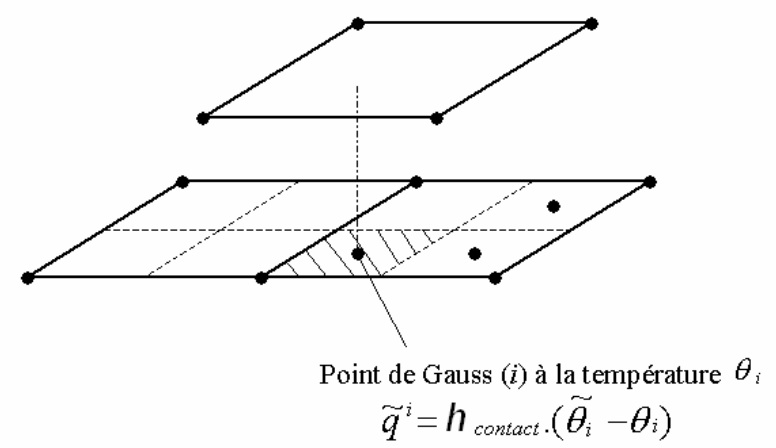

Figure 11. Schéma de principe du traitement du contact thermique tôle-support 
Cette modélisation localisée du contact n'assure pas globalement la conservation des flux de chaleur à l'interface qui peut être exprimée de la manière suivante :

$$
Q_{1}+Q_{2}=0
$$

avec $Q_{1}$ et $Q_{2}$ qui correspondent respectivement aux flux de chaleur entrant par les surfaces de contact $\partial \Omega_{1}$ et $\partial \Omega_{2}$ dans chacun des solides en contact $\Omega_{1}$ et $\Omega_{2}$ :

$$
Q_{1}=\int_{\partial \Omega_{1}} q \cdot d s \text { et } Q_{2}=\int_{\partial \Omega_{2}} q \cdot d s
$$

Etant donnée l'estimation de la densité de flux de chaleur en chaque point de Gauss, nous avons :

$$
\widetilde{Q}_{1}=\int_{\partial \Omega_{1}} \widetilde{q} \cdot d s \text { et } \widetilde{Q}_{2}=\int_{\partial \Omega_{2}} \widetilde{q} \cdot d s
$$

et généralement,

$$
\widetilde{Q}_{1}+\widetilde{Q}_{2} \neq 0
$$

Pour assurer la conservation des flux de chaleur, un coefficient de correction est introduit de la manière suivante :

$$
C \cdot \widetilde{Q}_{1}+\frac{1}{C} \cdot \widetilde{Q}_{2}=0
$$

On en déduit :

$$
C=\sqrt{-\frac{\widetilde{Q}_{2}}{\widetilde{Q}_{1}}}
$$

La densité de flux de chaleur peut alors être corrigée en chaque point de Gauss de chacune des surfaces $\partial \Omega_{1}$ et $\partial \Omega_{2}$ :

$$
q=C \cdot h_{\text {contact }} \cdot\left(\tilde{\theta}_{i}-\theta_{i}\right) \text { sur } \partial \Omega_{1}
$$




$$
q=\frac{1}{C} \cdot h_{\text {contact }} \cdot\left(\widetilde{\theta}_{i}-\theta_{i}\right) \text { sur } \partial \Omega_{2}
$$

\subsection{Résultats}

Les figures 12 et 13 présentent les températures obtenues pour une contrainte tangentielle de $50 \mathrm{MPa}$ à l'interface outil-tôle, une vitesse d'avance de $500 \mathrm{~mm} \cdot \mathrm{min}^{-1}$ et une vitesse de rotation de 1100 tr.min $^{-1}$. Cette configuration de soudage conduit à une température maximale de $465^{\circ} \mathrm{C}$ à l'arrière du pion. Cette température ne dépasse pas la température de fusion de l'alliage qui est supérieure à $500^{\circ} \mathrm{C}$. Par conséquent, la soudure est bien réalisée à l'état solide.

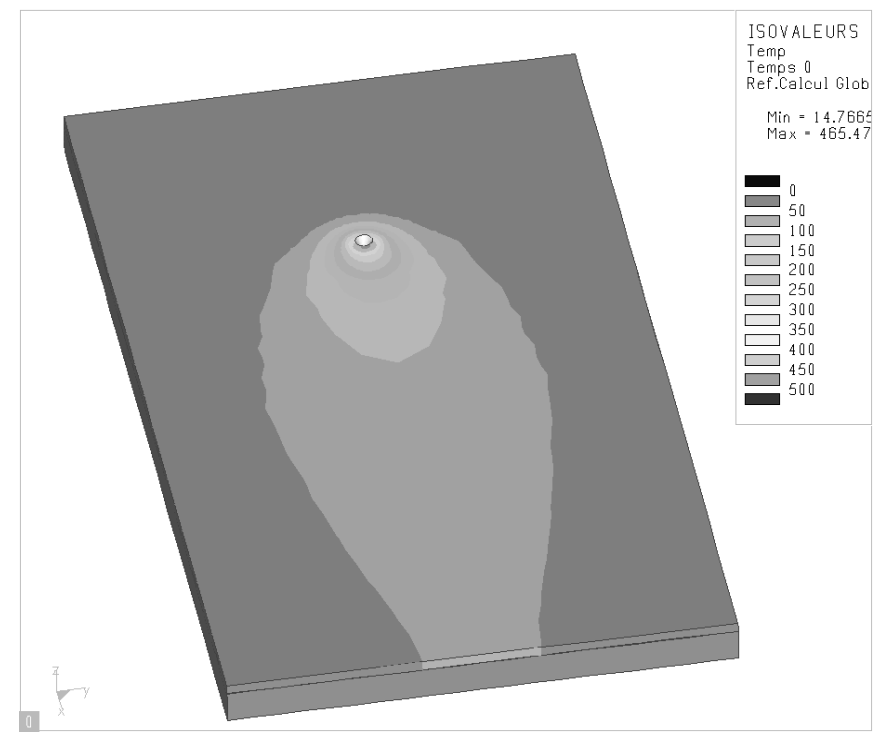

Figure 12. Champ des températures $\left({ }^{\circ} \mathrm{C}\right)$ calculé pour $\tau=50 M P a$, une vitesse d'avance de 500 mm. $\mathrm{min}^{-1}$ et une vitesse de rotation de 1 100tr. $\mathrm{min}^{-1}$

Comme le montre la figure 14, les élévations de température dans le support de soudage sont relativement faibles puisque la température maximale atteinte est de l'ordre de $34^{\circ} \mathrm{C}$. Plusieurs points peuvent expliquer cette faible montée en température. En particulier, la valeur de $h_{\text {contact }}$ est considérée constante et relativement faible or, elle dépend de nombreux paramètres qui ne sont pas pris en compte (température, pression de contact, etc.).

Par ailleurs, la figure 15 fait apparaître le caractère asymétrique du procédé FSW. Le tracé des lignes de courant montre clairement l'importance du malaxage que peut subir l'écoulement matériel au voisinage de l'outil. 
884 REMN - 16/2007. Modélisations numériques en mécanique

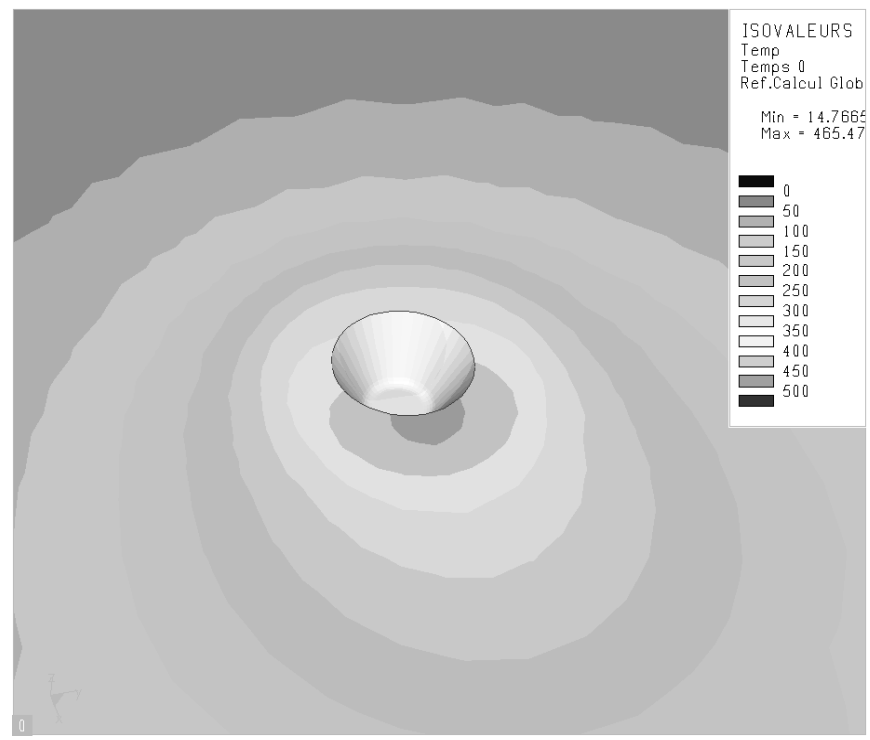

Figure 13. Champ des températures $\left({ }^{\circ} \mathrm{C}\right)$ au voisinage de l'outil pour $\tau=50 M P a$, une vitesse d'avance de $500 \mathrm{~mm} . \mathrm{min}^{-1}$ et une vitesse de rotation de $1100 \mathrm{tr}_{\mathrm{min}}^{-1}$

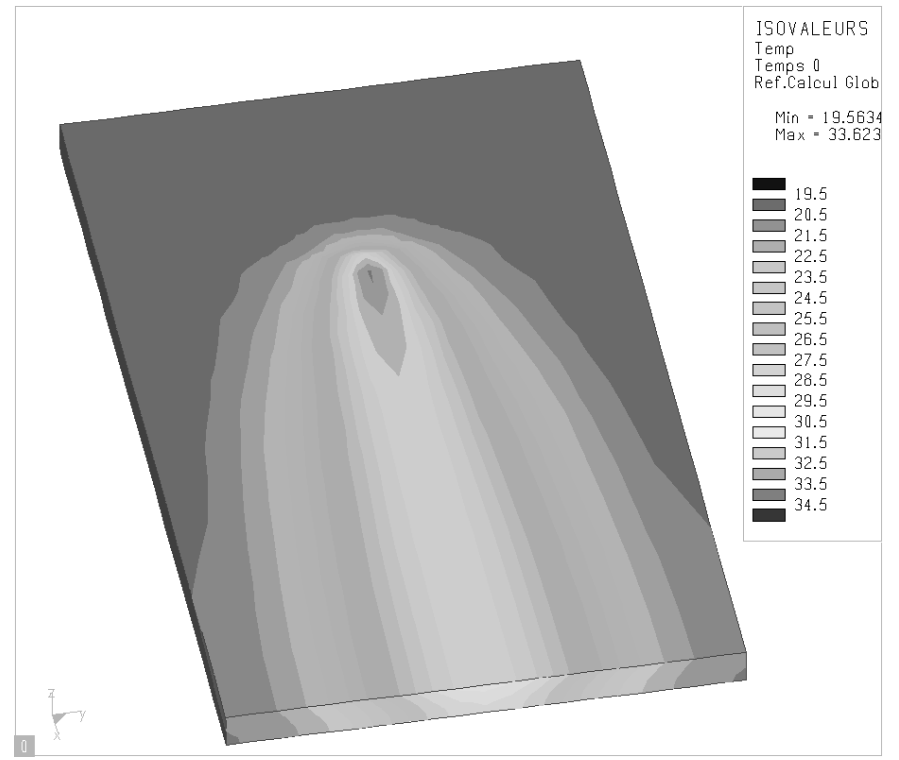

Figure 14. Champ des températures $\left({ }^{\circ} \mathrm{C}\right)$ calculé dans le support pour $\tau=50 \mathrm{MPa}$, une vitesse d'avance de $500 \mathrm{~mm} . \mathrm{min}^{-1}$ et une vitesse de rotation de $1100 \mathrm{tr} . \mathrm{min}^{-1}$ 


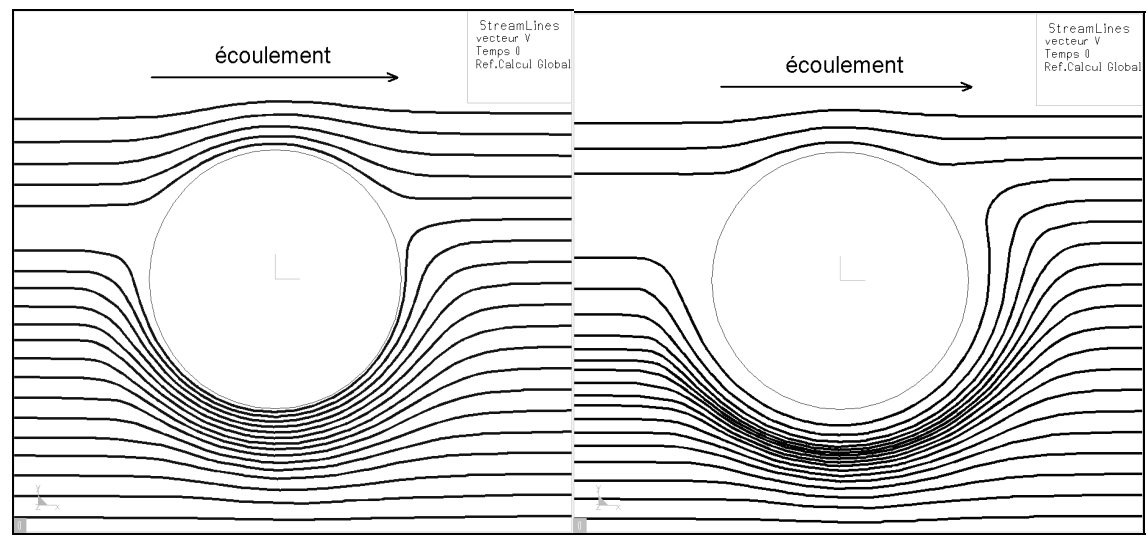

(a)

(b)

Figure 15. Lignes de courant au voisinage du pion dans le plan de l'épaulement

\section{Conclusion}

Le modèle numérique présenté permet de modéliser des écoulements visqueux couplés aux aspects thermiques dans une approche stationnaire. D'un point de vue numérique, les résultats obtenus sont tout à fait satisfaisants puisque pour l'exemple présenté qui comporte 11245 nœuds et 53920 éléments, les temps de calcul restent inférieurs à 2 heures sur un PC Pentium 4 à 3.4 Ghz équipé de 2 Go de RAM.

L'essentiel du travail à poursuivre consiste à modéliser les contraintes et distorsions résiduelles. De cette manière, la simulation du champ des températures associée à la modélisation des contraintes et distorsions résiduelles pourrait être appliquée à une approche de type local/global telle que celle proposée par Souloumiac et al. pour la modélisation du soudage de grandes structures minces (Michaleris et Debicarri, 1997 ; Souloumiac et al., 2001). Cette méthode consiste à ne simuler qu'une partie de la soudure sur un maillage 3D localisé afin de déterminer les déformations plastiques induites au voisinage du joint. Ces déformations peuvent ensuite être projetées sur le maillage complet de la structure constitué d'éléments finis coque pour accéder aux distorsions globales à partir d'une simple analyse élastique.

\section{Bibliographie}

Arnold D. N., Brezzi F., Fortin M., "A stable element for the Stokes equations", Calcolo., vol. 21, 1984, p. 337-344.

Babuska I., "The finite element method with Lagrangien multipliers", Numer Math., vol. 20, 1973, p. 179-192. 
Bastier A., Maitournam M. H., Dang Van K., «Approche eulérienne et intéraction fluidestructure pour la modélisation Du FrictionStir Welding ", $7^{e}$ colloque national en calcul des structures, Giens, 2005.

Bathe K.J., Finite Element Procedure, Prentice-Hall, 1996.

Bergheau J.-M., Fortunier R., Simulation numérique des transferts thermiques par éléments finis, Hermès Lavoisier, 2004.

Bergheau J.-M., Pont D., Leblond J.-B., "Three-dimensional simulation of a LASER surface treatment through steady state computation in the heat source comoving frame", Mechanical Effects of Welding, IUTAM Symposium Lulea (Sweden), 1991, edited by L. Karlsson, L.-E. Lindgren, M. Jonsson, 1992, Springer-Verlag, Berlin Heidelberg, p. 85-92.

Brezzi F., "On the existence, uniqueness and approximation of saddle-point problems arising from Lagrangien multipliers", RAIRO, Anal. Numer, R2, 1974, p. 129-151.

Brooks A. N., Hughes T. J.R., "Streamline Upwind/Petrov-Galerkin formulations for convection dominated flows with particular emphasis on the incompressible NavierStokes equations", Computer Methods in Applied Mechanics and Engineering, vol. 32, 1982, p. 199-259.

Colegrove P. A., Shercliff H. R., "Development of Trivex friction stir welding tool Part 2 three-dimensional flow modelling", Science and Techonoly of Welding and Joining, vol. 9 , n 4, 2004, p. 352-361.

Connolly B. J., Davenport A. J., Jariyaboon M., Padovani C., Ambat R., Williams S. W., Price D. A., Wescott A., Goodfellow C. J., Lee C. M., Proc. $5^{\text {th }}$ Int. Symp. on Friction Stir Welding, 2004.

Dang Van K., Inglebert G., Proix J. M., « Sur un nouvel algorithme de calcul des structures élastoplastiques en régime stationnaire ", $3^{e}$ colloque sur les tendances actuelles en calcul des structures, 1985.

Donne C. D., Lima E., Wegener J., Pyzalla A., Buslaps T., Proc. $3^{\text {rd }}$ Int. Symp. on Friction Stir Welding, 2001.

Feulvarch E., Robin V., Bergheau J. M., "Resistance spot welding simulation: a general formulation of electrothermal contact conditions", Journal of Materials Processing Technology, vol. 153-154, 2004, p. 436-441.

Feulvarch E., Rogeon P., Carre P., Robin V., Sibilia G., Bergheau J. M., "Resistance spot welding process: experimental and numerical modeling of the weld growth mechanisms with consideration of contact conditions", Numerical Heat Transfer, Part A, vol. 49, 2006, p. 345-367.

Feulvarch E, Modélisation numérique du soudage par friction-malaxage (Friction Stir Welding), Thèse de doctorat, Université Jean Monnet de Saint-Etienne, 2005.

Fourment L., Guerdoux S., Miles M., Nelson T., "Numerical Simulation of the Friction Stir Welding Process using both Lagrangian and Arbitrary Lagrangian Eulerian Formulations", Proc. $5^{\text {th }}$ Int. Symp. on Friction Stir Welding, 2004.

Gallais C., Joints soudés par friction malaxage d'alliages d'aluminium de la série 6XXX : caractérisation et modélisation, Thèse de doctorat, INPG, 2005. 
Gresho P. M., Sani R. L., Incompressible flow and the finite element method, Wiley, 2000.

Jaouen O., Modélisation tridimensionnelle par éléments finis pour l'analyse thermomécanique du refroidissement des pièces coulées, Thèse de doctorat, Ecole des Mines de Paris, 1998.

Jin Z., Cassada W. A., Cady C. M., Gray G. T., "Mechanical Response of AA7075 Aluminium Alloy over a Wide Range of Temperatures and Strain Rates", Materials Science Forum, Trans. Tech. Publications, vol. 331-337, 2000, p. 527-532.

Le Meur G., Etude de la condition de liaison thermique à une interface de contact solidesolide siège d'une dissipation par effet Joule : application au soudage par point, Thèse de doctorat, Ecole Polytechnique de l'université de Nantes, 2002.

Maitournam M. H., Formulation et résolution numérique des problèmes thermoviscoplastiques en régime permanent, Thèse de doctorat, Ecole Nationale des Ponts et Chaussées, 1989.

Michaleris P., Debiccari A., "Prediction of welding distortion", Welding Journal, vol. 76, 1997, p. 172-180.

Nguyen Q. S., Rahamian M., Mouvement permanent d'une fissure en milieu élastoplastique, Journée de Mécanique Appliquées, 1981.

Pascal R., Conraux P., Bergheau J. M., "Coupling between finite elements and boundary elements for the numerical simulation of induction heating processes using an harmonic balance method", IEEE Transactions on Magnetics, special CEFC issue, vol. 39, $\mathrm{n}^{\circ} 3$, 2003, p. 1535-1538.

Rajadhyaksha S. M. A., Michaleris P., "Optimization of thermal processes using an eulerian formulation and application in laser surface hardening", International Journal for Numerical Methods in Engineering, vol. 47, 2000, p. 1807-1823.

Reddy J. N., Gartling D. K., The finite element method in Heat transfer and fluid dynamics, CRC Press, 2000.

Reynolds A. P., "Friction Stir Welding of Aluminium Alloys", Handbook of aluminium: alloy production and materials manufacturing, 2003, p. 576.

Shanghvi J. Y., Michaleris P., "Thermo-elasto-plastic finite element analysis of quasi-state processes in eulerian reference frames", International Journal for Numerical Methods in Engineering, vol. 53, 2002, p. 1533-1556.

Shercliff H. R., Colegrove P. A., "Modelling of friction stir welding", Mathematical Modelling of Weld Phenomena, vol. 6, 2002, p. 927-974.

Souloumiac B., Boitout F., Bergheau J. M., "A new local/global approach for the modelling of welded component distortions”, Mathematical Modelling of Weld Phenomena, vol. 5, 2001.

SYSWELD ${ }^{\circledR}$, SYSWELD user’s manual, ESI Group, 2006.

Wagoner R. H., Chenot J.-L., Metal forming analysis, Cambridge University Press, 2001.

Zhang L., Michaleris P., "Investigation of Lagrangien and Eulerian finite element methods for modeling laser forming process", Finite element in analysis and design, vol. 40, 2004, p. $383-405$.

Zienkiewicz Z. O., Taylor R. L., « La méthode des elements finis », 1990. 
